\title{
MedSurv: a software application for creating, conducting and managing medical surveys and questionnaires Zachary S Ware ${ }^{1}$, Lisbeth A Selby ${ }^{2}$ and Jerzy W Jaromczyk*1
}

\author{
Address: ${ }^{1}$ Department of Computer Science, University of Kentucky, Lexington, KY 40506, USA and ${ }^{2}$ Department of Gastroenterology, University \\ of Kentucky, Lexington, KY 40506, USA \\ Email: Jerzy W Jaromczyk* - jurek@cs.uky.edu \\ * Corresponding author
}

from UT-ORNL-KBRIN Bioinformatics Summit 2008

Cadiz, KY, USA. 28-30 March 2008

Published: 8 July 2008

BMC Bioinformatics 2008, 9(Suppl 7):P3 doi:10.1 186/1471-2105-9-S7-P3

This abstract is available from: http://www.biomedcentral.com/I47I-2 105/9/S7/P3

(c) 2008 Ware et al; licensee BioMed Central Ltd.

\section{Background and solution}

MedSurv is a system designed for the rapid creation and maintenance of research surveys and questionnaires that does not require programmer intervention. MedSurv is built with medical surveys in mind and utilizes a groupbased permission control with additional security features to help ensure compliance with applicable healthcare regulations. MedSurv is designed as a module for DotNetNuke [1], an open source portal and content management system built with ASP.Net technology, and therefore can be deployed and managed as intranet, extranet, and web sites. At the same time, all data is stored at the researcher's institution to guarantee the required data privacy. Thanks to its built-in support for user authentication and user roles, there is no need to create such functionality from scratch. However, a group-based permissions system is added to MedSurv to support sufficient granularity for access control. Although from the data access point of view data storage acts as a relational table, MedSurv uses a solution that we call virtual tables. The premise behind such a solution is that the structure of the tables is itself stored in a set of relational tables within the database, essentially creating a miniature database within the database. This additional layer is transparent to the user and removes the need for any programming or database knowledge. At the same time it gives the user the flexibility of changing the survey at runtime. Unlike a traditional structure that may require database developer's involvement each time a survey is added or changed, with virtual tables there is very low developer and database adminis- tration need after launch. MedSurv allows for creating complex medical surveys and is, in particular, used to develop questionnaires for research driven data collection in the Department of Gastroenterology.

\section{Acknowledgements}

We acknowledge funding from the KBRIN-INBRE NIH 2 P20 RR-I648I, the National Center for Research Resources Institutional Development Award (IDeA) Program (subcontract from the University of Louisville).

\section{References}

I. DotNetNuke/DNN - Home (4.8.2) [http://www.dotnet 